\title{
La obligación empresarial de organizar la prevención de riesgos laborales: controversias aplicativas e interpretativas
}

The business obligation to organise the prevention of occupational risks: application and interpretation issues

\author{
Dr. Guillermo GARCÍA GONZÁLEZ \\ Universidad Internacional de La Rioja \\ guillermo.garcia@unir.net
}

\begin{abstract}
Resumen: La Ley 31/1995, de Prevención de Riesgos Laborales, impone como primera obligación empresarial el deber de implementar un modelo de organización preventiva a partir de las distintas posibilidades que la propia disposición normativa diseña. Desde la entrada en vigor de la norma, la organización de la prevención ha sido objeto de una especial atención doctrinal, teniendo en cuenta el carácter transversal de la materia, que imbrica la dinámica del conjunto de obligaciones preventivas empresariales. El presente artículo tiene como objetivo analizar la configuración jurídica de los distintos modelos de organización preventiva en el ámbito de la empresa, prestando singular atención a los problemas interpretativos y aplicativos que su ordenación normativa ha venido suscitado en la práctica
\end{abstract}

Abstract: Law 31/1995, on the Prevention of Occupational Risks, stipulates that it is the first responsibility of the company to implement a preventive organization model based on the different possibilities that the provision itself designs. Since the enactment of the regulation, the organisation of prevention has been the target of special doctrinal attention, taking into account the transversal nature of the subject, which interweaves with the dynamics of all the preventive business obligations. The objective of this article is to analyse the legal configuration of the different models of preventive organisation in the area of business, paying special attention to the interpretation and application problems that their normative organisation has aroused in practical terms.

Palabras clave: organización preventiva, prevención de riesgos laborales, seguridad y salud, servicios de prevención, trabajador designado. 
Keywords: preventive organization, prevention of occupational risks, health and safety, prevention services, appointed worker.

Sumario:

I. Introducción.

II. La libertad empresarial en la elección del modelo de organización preventiva: alcance y limitaciones.

III. La asunción personal por el empresario de la actividad preventiva: principales elementos controvertidos.

IV. La designación de trabajadores como modalidad preventiva: su indeterminada ordenación normativa.

V. Los servicios de prevención como instrumentos esenciales de integración de la actividad preventiva.

5.1. Los servicios de prevención propios y mancomunados.

5.2. Los servicios de prevención ajenos y su cuestionada capacidad de integración.

VI. A modo de reflexión final.

VII. Bibliografía.

Recibido: noviembre 2020.

Aceptado: enero 2021. 


\section{INTRODUCCIÓN}

La promulgación de la Ley 31/1995, de Prevención de Riesgos Laborales (LPRL), supuso un punto de inflexión en el derecho de la seguridad y salud en el trabajo en nuestro país, que ha experimentado desde entonces un desarrollo científico sin precedentes, pasando de ser una materia olvidada a "hiperestudiada"1. Esta norma y su intenso y extenso desarrollo reglamentario han dado lugar a un auténtico "subsector del ordenamiento jurídico", el derecho de la seguridad y salud laboral, entendido como una parcela jurídica "con entidad y características propias, obedientes a principios específicos y constitutivas de un sistema en sentido técnico-jurídico"2.

Entre las múltiples novedades introducidas, la LPRL impone a todas las empresas, como primera obligación, la de implementar un modelo de organización preventiva a partir de las distintas posibilidades que la propia norma establece (art. 30 LPRL) ${ }^{3}$. La configuración jurídica de estos modelos de gestión preventiva se hacía necesaria teniendo en cuenta la conformación del deber de seguridad empresarial como un deber complejo, integrado por un conjunto de obligaciones de alto contenido técnico ${ }^{4}$.

Así, y a partir de la LPRL, la organización de la actividad preventiva en la empresa se conforma como una obligación empresarial concreta, articulada a partir del diseño legal de distintos modelos organizativos. En este sentido, el capítulo IV LPRL, bajo la rúbrica "Servicios de Prevención", regula las distintas modalidades de organizar la prevención en el seno de las organizaciones productivas. La escueta ordenación legal es completada por el Real Decreto 39/1997, de 17 de enero, por el que se aprueba el Reglamento de los Servicios de Prevención (RSP), que desarrolla detalladamente el alcance y contenido de las distintas modalidades de organizar la prevención en la empresa (arts. 10 y ss).

\footnotetext{
${ }^{1}$ MONTOYA MELGAR, A., "Prólogo", en GUTIÉRREZ-SOLAR CALVO, B., El deber de seguridad y salud en el trabajo. Un estudio sobre su naturaleza juridica, Madrid 1999, p. 14.

${ }^{2}$ SEMPERE NAVARRO, A.V., "Aspectos jurídicos del Reglamento de Prevención de Riesgos Laborales”, en Aranzadi Social, 5 (1997) 571-592.

${ }^{3}$ Vid. MERCADER UGUINA, J.R., "La Ley de Prevención de Riesgos Laborales, veinte años después", en Revista de Información Laboral, 8 (2015) 21 y ss.

${ }^{4}$ GONZÁLEZ ORTEGA, S., y APARICIO TOVAR, J., Comentarios a la Ley 31/1995 de Prevención de Riesgos Laborales, Madrid 1996, p. 202.
} 
Con base en las normas precitadas, nuestro ordenamiento jurídico diseña cuatro modalidades de organizar la prevención: asunción por el empresario de la actividad preventiva, designación de uno o varios trabajadores de la empresa para desarrollar funciones preventivas, constitución de un servicio de prevención propio o mancomunado en la empresa, y el recurso a uno o varios servicios de prevención ajenos. Todas estas modalidades organizativas pueden considerarse en sentido amplio servicios de prevención, pues así deriva de la propia Directiva 89/391/CEE, que entiende a los servicios de prevención, no como el órgano en el que se integran los técnicos y desarrollan su actividad, sino como el conjunto de funciones ${ }^{5}$. Sin menoscabo de lo anterior, a lo largo del presente estudio se empleará el término servicios de prevención en sentido estricto para referir las específicas realidades que bajo esa denominación contempla nuestro ordenamiento jurídico (servicio de prevención propio, mancomunado y ajeno); y ello, con el objeto de facilitar la lectura y evitar confusiones terminológicas.

Teniendo en cuenta las anteriores consideraciones, el presente artículo tiene como objetivo analizar la configuración jurídica de los distintos modelos de organización preventiva en el ámbito de la empresa, prestando singular atención a los problemas interpretativos y aplicativos que su ordenación normativa ha venido suscitado en la práctica ${ }^{6}$.

\section{LA LIBERTAD EMPRESARIAL EN LA ELECCIÓN DEL MODELO DE ORGANIZACIÓN PREVENTIVA: ALCANCE Y LIMITACIONES}

La prevención de riesgos laborales entendida como conjunto de actividades o medidas adoptadas en todas las fases de actividad de la empresa con el fin de evitar o disminuir los riesgos derivados del trabajo, requiere una actuación empresarial que no se limite al mero cumplimiento formal de un conjunto predeterminado de deberes y obligaciones, sino que logre una integración

${ }^{5}$ MORENO SOLANA, A., El servicio de prevención en la empresa. Modalidades de organización de la Prevención de Riesgos Laborales, Cizur Menor 2016, p. 34. En análogos términos, vid. el completo estudio de MERCADER UGUINA, J.R., "El Reglamento de los Servicios de Prevención: Crónica de una norma largamente esperada", en Relaciones Laborales, 1 (1997) 1117-1142.

${ }^{6}$ Queda fuera del estudio, en consecuencia, la problemática que la organización de la prevención suscita en el ámbito de las Administraciones Públicas, que gozan en este ámbito de normativa específica ex arts. 3.1 y 31.1 LPRL y DA4 ${ }^{\text {a }}$ RSP. Sobre este punto, cfr. MERCADER UGUINA, J.R., "Ley de Prevención de Riesgos Laborales y Administraciones Públicas", en Relaciones Laborales, 1 (1996) 1137-1158, y GONZÁLEZ ORTEGA, S., "La aplicación de la Ley de Prevención de Riesgos Laborales a las administraciones públicas”, en Temas Laborales, 72 (2003) 13-33. Vid. STS, 24 de abril de 2001 (n rec. 3145/2000) y STSJ País Vasco, 14 de octubre de 2003 $\left(n^{\circ}\right.$ rec. 1796/2003). 
real y efectiva de la seguridad y salud laboral en todos los ámbitos de la organización productiva.

Atendiendo a la finalidad expuesta, y en el marco del deber general de seguridad empresarial, el art. 14.2 LPRL establece la obligación de las empresas de organizar la prevención de riesgos laborales, debiendo contar para ello con medios humanos y materiales suficientes en los términos establecidos por la LPRL y por su normativa complementaria. Esta obligación puede ser considerada como instrumental, estando al servicio de la sustancial contenida en el mismo precepto: "la adopción de cuantas medidas sean necesarias para la protección de la seguridad y la salud de los trabajadores" $"$.

Como ya ha sido apuntado, el empresario resulta obligado por la legislación vigente en materia de prevención de riesgos laborales a articular la organización de la prevención en el ámbito de la empresa, debiendo optar por alguna de las modalidades preventivas que son reguladas en los arts. 30 a 32 LPRL y 10 a 21 RSP: asunción por el empresario de la actividad preventiva, designación de uno o varios trabajadores de la empresa para desarrollar funciones preventivas, constitución de un servicio de prevención propio o mancomunado en la empresa, y el recurso a uno o varios servicios de prevención ajenos.

Las modalidades de organización preventiva dispuestas legalmente tienen carácter imperativo, por lo que la negociación colectiva no podrá introducir nuevas modalidades, sin perjuicio de que por medio de instrumentos colectivos se implementen opciones a favor de una u otra modalidad o se especifique su configuración interna, siempre que ello redunde en una mejora de las condiciones de seguridad y salud de los trabajadores.

El ordenamiento jurídico no considera cada una de las modalidades de organización preventiva excluyente de las demás, sino que implementa un sistema flexible, pudiendo el empresario combinar varias de ellas dentro del marco configurado legal y reglamentariamente ${ }^{8}$. Además, la opción del empresario por uno u otro modelo no condiciona a priori lo que ocurra en el futuro,

${ }^{7}$ GARCÍA GONZÁlEZ, G., y GARRIGUES GIMÉNEZ, A. (dirs.), Manual de Derecho de la Prevención de Riesgos Laborales, Valencia 2015, pp. 232-233, y GARATE CASTRO, J., "Organización de la prevención mediante la designación de uno o varios trabajadores", en Revista General de Derecho del Trabajo y de la Seguridad Social, 4 (2003) 1. Algún autor distingue las obligaciones de prevención de riesgos laborales entre las materiales o de ejecución y las orgánicas, correspondiendo a estas últimas la de organización de la prevención en el ámbito de la empresa. Cfr. OJEDA AVILÉS, A., "Claves interpretativas de la Ley 31/1995, sobre prevención de riesgos laborales", en La Ley, 1 (1997) 1182 y ss.

${ }^{8}$ STSJ Asturias, 19 de septiembre de 2014 ( $\mathrm{n}^{\mathrm{o}}$ rec. 1708/2014). 
pudiendo ser esta opción revisada o modificada posteriormente en atención a las circunstancias concurrentes y a la libertad empresarial ${ }^{9}$.

Sin menoscabo de la flexibilidad apuntada, la elección del sistema de organizar la prevención en el ámbito de la empresa no queda siempre a la libre disposición del empresario, ya que existen determinados límites normativos que deben ser respetados en función de la actividad empresarial y del número de trabajadores con que cuente la empresa. Así, y como posteriormente se analizará, en ocasiones el ordenamiento jurídico no permite que el empresario pueda asumir personalmente la prevención (en las empresas de un determinado volumen de trabajadores o con actividades especialmente peligrosas), y en otros casos exige a la empresa la constitución de un servicio de prevención propio (en función del número de trabajadores y del tipo de actividad que desarrolle) ${ }^{10}$.

Junto con las limitaciones legales y reglamentarias referidas, el empresario tiene otro condicionante -si bien de eficacia limitada- en su proceso decisorio del modelo de organización preventiva a implantar: la necesaria intervención de los trabajadores a través de sus órganos de representación en la conformación definitiva de la decisión empresarial sobre la materia. En este sentido, el empresario debe consultar con los representantes de los trabajadores el modelo organizativo de la prevención de riesgos laborales a implantar en el ámbito de la empresa.

El derecho que tienen los trabajadores a ser consultados en materia de prevención de riesgos laborales queda regulado fundamentalmente en los arts. 18.2, 33, 38 y 39 LPRL, así como en los arts. 16.2, 21.1 y 30.5 RSP. La consulta debe realizarse a los trabajadores a través de sus representantes, siempre que la empresa cuente con ellos.

A estos efectos, por representantes de los trabajadores deben entenderse tanto los delegados de personal, como los comités de empresa y los delegados sindicales ${ }^{11}$; si bien, parece lógico que con carácter prioritario el empresario formule las consultas a los delegados de prevención en cuanto órganos de representación especializados en materia preventiva ${ }^{12}$. Así lo entiende el

\footnotetext{
${ }^{9}$ SEMPERE NAVARRO, A.V., "Aspectos jurídicos del Reglamento de Prevención de Riesgos Laborales", o.c., 571-592.

10 SSTS, 24 de abril de 2001 ( $\mathrm{n}^{\mathrm{o}}$ rec. 3145/2000) y 3 de noviembre de 2005 ( $^{\mathrm{o}}$ rec.154/2004).

${ }^{11}$ STS, 6 de mayo de 1998 ( $n^{\circ}$ rec. 3301/1997).

12 ROMERAL HERNÁNDEZ, J., "Derechos de información, consulta y crédito horario del delegado de prevención a la luz de la jurisprudencia", en Revista Española de Derecho del Trabajo, 209 (2018) 23 y ss. Cfr. STS, 16 de febrero de 2016 ( ${ }^{\circ}$ rec. 250/2014).
} 
legislador que, en el art. $36.1 \mathrm{c})$ LPRL, establece como una de las competencias de los delegados de prevención la de ser consultados por el empresario, con carácter previo a su ejecución, acerca de las materias recogidas por el art. 33 LPRL. En defecto de delegados de prevención, la consulta deberá plantearse a los representantes unitarios o sindicales que existan en la empresa. En ausencia de representantes, el empresario deberá consultar directamente a los propios trabajadores individualmente considerados, rigiendo en este último caso lo dispuesto en los arts. 77 y ss. del Real Decreto Legislativo 2/2015, de 23 de octubre, por el que se aprueba el texto refundido de la Ley del Estatuto de los Trabajadores (TRET).

En el supuesto concreto del modelo organizativo, la obligación de consulta con la representación de los trabajadores deriva del art. 33.1 b) LPRL y de los arts. 16.2 y 21.2 RSP. El primero de estos preceptos impone al empresario la obligación de consultar con la representación de los trabajadores "la organización y desarrollo de las actividades de protección de la salud y prevención de los riesgos profesionales en la empresa, incluida la designación de los trabajadores encargados de dichas actividades o el recurso a un servicio de prevención externo". El art. 16.2 RSP reitera lo dispuesto en el art. 33.1 b) LPRL, exigiendo la consulta previa del empresario a los representantes de los trabajadores antes de adoptar la "decisión de concertar la actividad preventiva con uno o varios servicios de prevención ajenos", reproduciendo dicha obligación el art. 21.2 RSP en lo que atañe a los servicios de prevención mancomunados.

Al igual que el resto de consultas en materia preventiva, esta consulta tiene carácter preceptivo aunque no vincula la decisión última del empresario, lo que la dota de una eficacia muy limitada ${ }^{13}$.

Si el empresario incumpliera su obligación de consulta sobre una decisión de trascendencia preventiva en la empresa, dicho incumplimiento supondrá una infracción administrativa de carácter grave ex art. 12.11 del Real Decreto Legislativo 5/2000, de 4 de agosto, por el que se aprueba el texto refundido de la Ley sobre Infracciones y Sanciones en el Orden Social (LISOS), resultando controvertido determinar si de dicho incumplimiento se podrá o no derivar la nulidad de la decisión adoptada sin el trámite de consulta.

En relación con lo anterior, un sector doctrinal entiende que la inobservancia de la obligación de consulta no determina por sí misma la nulidad de la medida adoptada por el empresario, constituyendo esta obligación un mero control

\footnotetext{
${ }^{13}$ SAN, 1 de febrero de 1999 (n ${ }^{\circ}$ rec. 187/1998).
} 
externo de la toma de decisiones empresarial ${ }^{14}$. Por el contrario, otro sector de la doctrina considera que la obligación de consulta se constituye como un trámite interno que ha de incorporarse en el proceso de adopción de las decisiones empresariales en materia de prevención, con lo que su inobservancia daría lugar a la irregularidad de dicho proceso decisional y, por tanto, determina la nulidad de la decisión ${ }^{15}$.

La consulta tiene sentido incluso en aquellos casos en los que la empresa debe necesariamente optar por el servicio de prevención propio atendiendo al volumen de plantilla y a su actividad; y ello, porque también en este supuesto la consulta a los trabajadores puede abarcar aspectos aparentemente adjetivos (composición del servicio de prevención propio, especialidades asumidas, distribución territorial, componentes, formación, medios materiales, entre otros) que son en ocasiones más relevantes que el propio modelo en sí mismo considerado ${ }^{16}$.

La LPRL no establece reglas estrictas sobre cuándo ha de realizarse la consulta a los trabajadores, previendo simplemente que se ha de formalizar "con la debida antelación" (art. 33.1 LPRL), o en el caso de los delegados de prevención "con carácter previo a su ejecución" (art. 36.1.c) LPRL). Los tribunales han interpretado que la expresión "con la debida antelación" conlleva la obligación de consultar a los trabajadores previamente a la instauración efectiva de cualquier acción o medida con implicaciones en seguridad y salud laboral ${ }^{17}$.

Respecto a la forma de llevar a cabo la consulta a los trabajadores, la LPRL tampoco señala nada al respecto, siendo lo conveniente que se realice tanto la petición como la contestación por escrito, tal como sí que se exige para los delegados de prevención cuando contestan a la consulta, que deberán hacerlo a través de un informe que se elaborará en un plazo de quince días, o en el tiempo imprescindible cuando se trate de adoptar medidas dirigidas a prevenir riesgos

${ }^{14}$ En este sentido, MARTÍNEZ ASO, M., La eficacia de la protección del derecho a la seguridad y salud en el trabajo de los trabajadores extranjeros, Girona 2013, pp. 154-155. Disponible en: https://www.tdx.cat/bitstream/handle/10803/124038/tmma.pdf;sequence=2 (fecha de consulta: 2 de octubre de 2020).

${ }^{15}$ ROMERAL HERNÁNDEZ, J., "Derechos de información, consulta y crédito horario del delegado de prevención a la luz de la jurisprudencia", o.c., 23 y ss., y SEMPERE NAVARRO, A.V., "Aspectos laborales de las leyes sobre libre acceso a los servicios", en Aranzadi Doctrinal, 2 (2010), 176. Cfr. STS, 16 de febrero de 2016 ( $n^{\circ}$ rec. 250/2014).

${ }^{16}$ En este sentido, GONZÁLEZ ORTEGA, S., "La organización de la prevención por el empresario: los servicios de prevención”, en Temas Laborales, 50 (1999), 42.

${ }^{17}$ STSJ Cantabria, 13 de julio de 2001 ( ${ }^{\circ}$ rec. 138/2001). 
inminentes (art. 36.3 LPRL) ${ }^{18}$. Esta diferencia de tratamiento o especificación respecto a la contestación de los delegados de prevención se basa en que, si el empresario no acoge las propuestas de estos, deberá fundamentar su decisión, y en el caso de que acaezca un accidente por la inobservancia de esta propuesta se graduará la sanción como consecuencia de ello (art. 39.1.g) LISOS).

La obligación de consulta respecto al modelo organizativo se encuentra directamente conectada con lo dispuesto en el art. 39.1 a) LPRL, que recoge entre las competencias del comité de seguridad y salud debatir "antes de su puesta en práctica y en lo referente a su incidencia en la prevención de riesgos, la elección de la modalidad organizativa de la empresa y, en su caso, la gestión realizada por las entidades especializadas con las que la empresa hubiera concertado la realización de actividades preventivas". En desarrollo de este precepto, el art. 16.2 RSP dispone que en el caso de que la empresa quiera adoptar la decisión de concertar la actividad preventiva con uno o varios servicios de prevención ajenos, los criterios a tener en cuenta para la selección de la entidad y las características técnicas del concierto se debatirán y, en su caso, se acordarán en el seno del comité de seguridad y salud. Análoga fórmula establece el art. 21.2 RSP respecto a los servicios de prevención mancomunados, debiendo debatirse y, en su caso, acordarse en el seno de cada uno de los comités de seguridad y salud de las empresas afectadas las condiciones en que debe desarrollarse dicho servicio (art. 21.2 RSP).

Por último, y aunque las consultas evacuadas por el comité de seguridad y salud no resultan vinculantes, cabe la posibilidad de que las partes pacten este carácter para las mismas ${ }^{19}$.

Hechas las precisiones que anteceden, se examinan a continuación los diferentes modos de organizar la prevención con los que cuenta el empresario, haciendo especial referencia a las principales inconcreciones normativas y al modo en que las mismas han sido integradas por la profusa labor doctrinal y, en menor medida, por la tarea de jueces y tribunales.

\footnotetext{
18 ROMERAL HERNÁNDEZ, J., "Derechos de información, consulta y crédito horario del delegado de prevención a la luz de la jurisprudencia”, o.c., 23 y ss. Cfr. STS, 16 de febrero de 2016 (n ${ }^{\circ}$ rec. 250/2014).

19 SSTS, 3 de noviembre de 2005 ( $n^{\circ}$ rec. 154/2004) y 11 de marzo de 2013 ( $\mathrm{n}^{\mathrm{o}}$ rec. 70/2012).
} 


\section{LA ASUNCIÓN PERSONAL POR EL EMPRESARIO DE LA ACTIVIDAD PREVENTIVA: PRINCIPALES ELEMENTOS CON- TROVERTIDOS}

Entre las diferentes modalidades de gestión preventiva, el art. 30.5 LPRL y el art. 11 RSP establecen que el empresario podrá asumir personalmente la actividad preventiva de su empresa, siempre que concurran determinadas condiciones en su organización productiva. Se trata de una forma de organización de la prevención excepcional, por cuanto la misma solo resulta aplicable a una concreta tipología de empresas, constituyendo la forma más básica o "rudimentaria" de gestionar la seguridad y salud laboral ${ }^{20}$.

De acuerdo con los preceptos más arriba referidos, podrán acogerse a esta modalidad organizativa las empresas en las que concurran las siguientes condiciones:

a) Que la empresa cuente con hasta 10 trabajadores o hasta 25 trabajadores, siempre que en este último caso tenga un único centro de trabajo. A estos efectos, la unidad de cálculo la constituye la empresa y no el centro de trabajo $^{21}$. Deberán computarse todos los trabajadores existentes en la plantilla con independencia del tipo de contrato que tengan o de la jornada laboral que desarrollen, tomando como referencia el periodo de un año ${ }^{22}$.

b) Que el empresario desarrolle su actividad de forma habitual en el centro de trabajo, y ello con independencia de que su trabajo sea manual o directivo. No se considerará cumplido este requisito si el empresario tiene una presencia esporádica o cíclica en el centro de trabajo, pues su presencia habitual en el mismo es un requisito indispensable ${ }^{23}$. En el caso de que la empresa tuviera más de un centro de trabajo, será preciso que el empresario acuda habitualmente a todos los centros de trabajo cuya gestión preventiva asuma personalmente ${ }^{24}$. Por otra parte, y aunque del literal de la norma parece deducirse que el empresario debe ser persona fisica para poder optar

${ }^{20}$ STS, 24 de abril de 2001 ( $n^{\circ}$ rec. 3145/2000).

${ }^{21}$ STSJ Madrid, contencioso-administrativo, 21 de octubre de 2005 ( $\mathrm{n}^{\circ} \mathrm{rec} .542 / 2002$ ).

${ }^{22}$ SALA FRANCO, T., Derecho de la Prevención de Riesgos Laborales, Valencia 2011, p. 158, y MORENO SOLANA, A., El servicio de prevención en la empresa. Modalidades de organización de la Prevención de Riesgos Laborales, o.c., pp. 273-281. Cfr. STSJ Madrid, contencioso-administrativo, 23 de diciembre de 2004 ( $n^{\circ}$ rec. 932/2000). En contra, optando por un cómputo diferenciado en función de la duración contractual y del tipo de jornada, vid. LUQUE PARRA, M., La organización de la prevención en la empresa, Valencia 2001, pp. 61-62.

${ }^{23}$ GARCÍA NINET, J.I. (dir.), Lecciones sobre la Ley de Prevención de Riesgos Laborales, Castelló de la Plana 1997, p. 152.

${ }^{24}$ En este sentido, LOUSADA AROCHENA, J.F., "Prevención de riesgos laborales en pequeñas y medianas empresas", en Revista Española de Derecho del Trabajo, 171 (2014) 245 y ss. 
por este modelo organizativo, nada parece oponerse a que esta modalidad fuera acogida por un empresario societario, siempre que la sociedad "tenga una fuerte base personalista y los socios participen activa y presencialmente en la gestión de la empresa"25.

c) El empresario ha de contar con la capacidad necesaria en función de los riesgos a que estén expuestos los trabajadores y la peligrosidad de las actividades, de acuerdo con lo determinado en el capítulo VI RSP, y que, por tanto, nunca podrá ser inferior a la formación de nivel básico ${ }^{26}$. Con todo, este nivel no habilita al empresario para realizar todas las acciones preventivas incluidas en el deber de seguridad empresarial, como es el caso de la obligación de formación ex art. 19 LPRL, que no se encuentra incluida entre las funciones atribuidas al técnico de prevención de nivel básico por el art. $35 \mathrm{RSP}^{27}$.

d) Que la actividad desarrollada no sea de las recogidas en el anexo I RSP. El anexo I RSP contiene un listado detallado de actividades especialmente peligrosas, entre las que se encuentran las siguientes: trabajos con exposición a radiaciones ionizantes; trabajos con exposición a agentes tóxicos y muy tóxicos, y en particular a agentes cancerígenos, mutagénicos o tóxicos para la reproducción; actividades en que intervienen productos químicos de alto riesgo; trabajos con exposición a agentes biológicos de los grupos 3 y 4; actividades de fabricación, manipulación y utilización de explosivos, incluidos los artículos pirotécnicos y otros objetos o instrumentos que contengan explosivos; trabajos propios de minería a cielo abierto y de interior, y sondeos en superficie terrestre o en plataformas marinas; actividades en inmersión bajo el agua; actividades en obras de construcción,

${ }^{25}$ MOLINA NAVARRETE, C., "Servicios de Prevención", en AAVV, Comentario a la Ley de Prevención de Riesgos Laborales y sus desarrollos reglamentarios, Granada 2004, p. 325. En idéntico sentido, SALA FRANCO, T., Derecho de la Prevención de Riesgos Laborales, o.c., p. 155, y MORENO SOLANA, A., El servicio de prevención en la empresa. Modalidades de organización de la Prevención de Riesgos Laborales, o.c., p. 462. En contra de esta postura se manifiesta gran parte de la doctrina. Cfr. GARRIGUES GIMÉNEZ, A. (dir.), Derecho de la prevención de riesgos laborales, Albacete 2009, p. 298, GONZÁLEZ ORTEGA, S., y APARICIO TOVAR, J., Comentarios a la Ley 31/1995 de Prevención de Riesgos Laborales, o.c., p. 197, y VIDA SORIA, J. (dir.), Manual para la formación en prevención de riesgos laborales, Valladolid 2003, p. 194.

${ }^{26}$ STSJ Madrid, contencioso-administrativo, 14 de mayo de 2004 ( $\left.\mathrm{n}^{\circ} \mathrm{rec} .43 / 2002\right)$. Cfr. FERNÁNDEZ MARCOS, L., Comentarios a la Ley de Prevención de Riesgos Laborales y normativa complementaria, Madrid 2001, pp. 162-163. Un sector doctrinal, si bien minoritario, entiende que la capacidad preventiva en el empresario debe presumirse siempre que la actividad de la empresa no se incardine en el anexo I RSP. Vid. MONTOYA MELGAR, A., y PIZÁ GRANADOS, J., Curso de seguridad y salud en el trabajo, Madrid 2000, p. 89.

${ }^{27}$ VALLEJO DACOSTA, R. y LAFUENTE PASTOR, V.P., Marco jurídico de la seguridad $y$ salud en el trabajo, Zaragoza 2016, p. 175 
excavación, movimientos de tierras y túneles, con riesgo de caída de altura o sepultamiento; actividades en la industria siderúrgica y en la construcción naval; producción de gases comprimidos, licuados o disueltos o utilización significativa de los mismos; trabajos que produzcan concentraciones elevadas de polvo silíceo; y trabajos con riesgos eléctricos en alta tensión.

Este listado de actividades debe ser entendido de forma estricta conforme al literal de la norma, sin que resulte posible realizar una interpretación extensiva incluyendo actividades que, aunque análogas, no se encuentren relacionadas en el anexo I RSP ${ }^{28}$. Resulta dudoso si se puede acoger a esta modalidad organizativa aquella empresa en la que solo alguna fase de su proceso productivo se encuadre en el anexo I RSP; la doctrina mayoritaria mantiene que en estos casos no cabe la asunción personal de la prevención por el empresario ${ }^{29}$.

e) No cabrá la asunción de la vigilancia de la salud por parte del empresario, debiendo cubrirse esta actividad, así como todas aquellas especialidades preventivas no asumidas personalmente por este, por alguna de las modalidades preventivas contempladas legalmente (art. 11.2 RSP). La imposibilidad de que el empresario pueda asumir funciones relativas a la vigilancia de la salud constituye un límite de derecho necesario y se anuda a la necesaria tutela del derecho a la intimidad y a la garantía de la confidencialidad de los datos sanitarios del trabajador.

Concurriendo todos los requisitos necesarios, la asunción por el empresario de la prevención resultará facultativa, pudiendo este optar por otra modalidad organizativa $^{30}$.

\section{LA DESIGNACIÓN DE TRABAJADORES COMO MODALIDAD PREVENTIVA: SU INDETERMINADA ORDENACIÓN NORMA- TIVA}

El art. 30 LPRL y los arts. 12 y 13 RSP establecen como modalidad de organización preventiva la designación de trabajadores. Se ha considerado esta modalidad organizativa como general y arquetípica, sirviendo de referencia a las restantes ${ }^{31}$.

\footnotetext{
${ }^{28}$ STSJ Navarra, 2 de octubre de 2010 (nº rec. 214/2010).

${ }^{29}$ SALA FRANCO, T., Derecho de la Prevención de Riesgos Laborales, o.c., p. 155.

${ }^{30}$ Cfr. SAN, 12 de julio de 2004 ( $n^{\circ}$ rec. 31/2004).

${ }^{31}$ GARATE CASTRO, J., "Organización de la prevención mediante la designación de uno o varios trabajadores", o.c., 3. En análogos términos, FERNÁNDEZ MARCOS, L., "El contenido 
Pese a que de la Directiva 89/391/CEE y del art. 31.1 LPRL se deduce que esta modalidad organizativa ha de ser la de preferente implantación en la empresa, la amplia libertad con la que goza el empresario para organizar la prevención de riesgos laborales ha situado a esta modalidad preventiva, en la práctica, en una situación secundaria respecto a los servicios de prevención, especialmente los ajenos ${ }^{32}$.

En el marco de su potestad organizativa, el empresario podrá recurrir a la designación de trabajadores como modalidad de organización preventiva en cualquier caso, salvo que resulte obligado por ley a constituir un servicio de prevención propio. La designación de trabajadores es, por tanto, facultativa para el empresario, que podrá organizar la prevención a través de cualquier otra modalidad; extremo que expresamente recoge el art. 12.2 RSP.

La designación de trabajadores debe entenderse tanto en el sentido de asignar funciones preventivas a un trabajador ya existente en la plantilla, como a la posibilidad que tiene el empresario de contratar a uno o varios trabajadores para la realización de las funciones preventivas en el ámbito de la empresa ${ }^{33}$.

El marco jurídico de los trabajadores designados es impreciso e indeterminado. A excepción del régimen de garantías que establece el art. 30.4 LPRL, la ordenación de esta modalidad preventiva viene caracterizada por su indefinición; indefinición que ha sido subsanada, siquiera parcialmente, por la labor de integración desarrollada por la doctrina y, en menor medida, por la jurisprudencia.

De acuerdo con el art. 30.2 LPRL, los trabajadores designados deberán tener la capacidad necesaria, disponer del tiempo y de los medios precisos y ser suficientes en número, teniendo en cuenta el tamaño de la empresa, así como los riesgos a que están expuestos los trabajadores y su distribución en la misma ${ }^{34}$. La simple lectura del precepto dibuja un escenario de inseguridad jurídica, al concurrir en la ordenación normativa diversos conceptos jurídicos indeterminados -"capacidad necesaria", "disponer del tiempo y de los medios precisos" y "ser suficientes en número"- que requieren ser precisados.

Siendo consciente de la inconcreta configuración legal en esta materia, el legislador atribuye su específica delimitación a la autonomía colectiva. En este sentido, la DA $7^{\mathrm{a}}$ RSP faculta a la negociación colectiva para el establecimiento de

de la Ley de Prevención de Riesgos Laborales: Puntos críticos", en Actualidad Laboral, 3 (1995) 817-841.

${ }^{32}$ Vid. INSST, Encuesta Nacional de Gestión de Riesgos Laborales en las Empresas. ESENER-2 España, Madrid 2015.

${ }^{33}$ SALA FRANCO, T., Derecho de la Prevención de Riesgos Laborales, o.c., p. 152.

${ }^{34}$ Cfr. STSJ Extremadura, contencioso-administrativo, 28 de febrero de 2005 ( $\mathrm{n}^{\circ} \mathrm{rec} .394 / 2003$ ). 
criterios que determinen el número de trabajadores designados por el empresario para llevar a cabo actividades de prevención, así como para concretar el tiempo y los medios de que deban disponer los trabajadores designados para el desempeño de su actividad.

Pese al esencial papel que está llamada a desempeñar en este ámbito, la autonomía colectiva apenas ha demostrado interés por complementar las lagunas normativas del régimen jurídico de los trabajadores designados. Los escasos instrumentos colectivos que ordenan esta materia "constituyen meras remisiones a la normativa legal y reglamentaria o formulaciones genéricas y, normalmente, previstas ya en la ley, reiterando, incluso, sus idénticas expresiones y conceptos indeterminados, lo que tiene menos justificación. Ello nos sitúa ante un círculo vicioso de indeterminación legal y convencional al que, no sin razón, se imputa buena parte de las deficiencias de nuestro modelo preventivo" 35 .

Si no existiera disposición convencional que regulara esta materia, y ante el silencio legal en este punto, será el empresario el que deba determinar en cada supuesto concreto el número de trabajadores designados necesarios y los medios con los que deben contar en función de la actividad de la empresa, del volumen de plantilla y de otras circunstancias que puedan tener incidencia en materia de seguridad y salud laboral.

Pese a que la normativa no señala nada al respecto, parece recomendable que el trabajador designado forme parte de la plantilla fija de la empresa y que cuente con una antigüedad suficiente para conocer los procesos y trabajos desarrollados en la organización productiva ${ }^{36}$. Además, ha de tenerse en cuenta que las funciones encomendadas al trabajador designado forman parte de la actividad permanente de la empresa, y que solo excepcionalmente podrá concurrir causa que ampare una contratación temporal del trabajador designado ${ }^{37}$.

La dedicación a funciones preventivas de los trabajadores designados puede ser tanto a tiempo completo como a tiempo parcial. Con el fin de dar cumplimiento a sus funciones preventivas, el empresario deberá facilitar a los trabajadores designados el acceso a la información y documentación en los

35 TUDELA CAMBRONERO, G., y VALDEOLIVAS GARCÍA, Y., La seguridad y la salud laboral en la negociación colectiva, Madrid 2009, pp. 400.

${ }^{36}$ GARCÍA GONZÁLEZ, G., y GARRIGUES GIMÉNEZ, A. (dirs.), Manual de Derecho de la Prevención de Riesgos Laborales, o.c., pp. 242-243.

37 TOSCANI GIMÉNEZ, D., "Obligaciones y derechos de los trabajadores con funciones preventivas”, en Gestión Práctica de Riesgos Laborales, 87 (2011) 31. 
términos establecidos en los arts. 18 y 23 LPRL, pues sin esos datos difícilmente podrán cumplir sus obligaciones preventivas ${ }^{38}$.

Respecto a la capacitación preventiva con la que han de contar los trabajadores designados, la misma dependerá de las funciones que les sean atribuidas de conformidad con lo dispuesto en el capítulo VI RSP: funciones de nivel básico, intermedio y superior. La capacidad que se exige a los trabajadores designados está directamente relacionada con su formación preventiva, sin que quepa asimilar dicha formación con la experiencia laboral general o específica en materia de prevención de riesgos laborales ${ }^{39}$. La formación requerida también variará en atención al tipo de empresa, actividad desarrollada, centros de trabajo, y complejidad y peligrosidad del proceso productivo, entre otros factores ${ }^{40}$.

El impreciso régimen jurídico de los trabajadores designados y la exigua labor de integración que ha desarrollado la negociación colectiva en esta materia, han dibujado un escenario de inseguridad jurídica que ha desincentivado el empleo de esta modalidad organizativa. De este modo, la teórica prioridad que esta forma de organización preventiva había de tener como modo de gestionar la actividad preventiva, se ha convertido en la práctica en un empleo residual o complementario respecto a los servicios de prevención externos. Una mayor precisión normativa, una negociación colectiva más comprometida y la implementación de políticas públicas que incentiven la designación de trabajadores como modalidad de organización preventiva, se presentan como instrumentos necesarios para poder revertir la relativa irrelevancia que en la práctica empresarial tiene esta forma de gestión preventiva.

\section{LOS SERVICIOS DE PREVENCIÓN COMO INSTRUMENTOS ESENCIALES DE INTEGRACIÓN DE LA ACTIVIDAD PRE- VENTIVA}

Entre las modalidades de organización preventiva de las que dispone el empresario, especial mención merecen los servicios de prevención, al presentarse como la fórmula organizativa mayoritariamente empleada por las organizaciones productivas. Se entiende por servicio de prevención el conjunto de medios

\footnotetext{
${ }^{38}$ GONZÁlEZ ORTEGA, S., y APARICIO TOVAR, J., Comentarios a la Ley 31/1995 de Prevención de Riesgos Laborales, o.c., p. 197.

${ }^{39}$ Vid. STSJ Murcia, contencioso-administrativo, 7 de marzo de 2008 ( $n^{\circ}$ rec. 1508/2003) y STSJ Castilla y León, Burgos, 23 de diciembre de 2011 ( $\mathrm{n}^{\mathrm{o}}$ rec. 221/2011).

${ }^{40}$ Cfr. FERNÁNDEZ MARCOS, L., Comentarios a la Ley de Prevención de Riesgos Laborales y normativa complementaria, o.c., p. 166.
} 
humanos y materiales necesarios para realizar las actividades preventivas a fin de garantizar la adecuada protección de la seguridad y la salud de los trabajadores, asesorando y asistiendo para ello al empresario, a los trabajadores y a sus representantes y a los órganos de representación especializados (art. 31.2 LPRL). Se refiere en este punto el legislador al sentido estricto del servicio de prevención, en cuanto a un concreto tipo de organización para el desarrollo de las funciones preventivas.

El servicio de prevención tendrá carácter interdisciplinar, debiendo contar con medios apropiados para cumplir sus funciones ${ }^{41}$. Para ello, la formación, especialidad, capacitación, dedicación y número de componentes de estos servicios, así como sus recursos técnicos, deberán ser suficientes y adecuados a las actividades preventivas a desarrollar, en función del tamaño de la empresa, los tipos de riesgos a los que puedan encontrarse expuestos los trabajadores y su distribución en la empresa.

Las funciones de los servicios de prevención vienen recogidas en el art. 31.3 LPRL y consisten en prestar a la empresa el asesoramiento y apoyo que precise en materia preventiva y, en particular, en lo referente a:

- Diseño, implantación y aplicación de un plan de prevención de riesgos laborales que permita la integración de la prevención en la empresa.

- Evaluación de los factores de riesgo que puedan afectar a la seguridad y la salud de los trabajadores en los términos previstos en el art. 16 LPRL.

- Planificación de la actividad preventiva, determinación de las prioridades en la adopción de las medidas preventivas y vigilancia de su eficacia.

- Información y formación de los trabajadores, en los términos previstos en los arts. 18 y 19 LPRL.

- Prestación de los primeros auxilios y planes de emergencia.

- Vigilancia de la salud de los trabajadores en relación con los riesgos derivados del trabajo.

Existen tres tipos de servicios de prevención: los propios, los mancomunados y los ajenos. Cada uno de estos tipos de servicios de prevención, aun teniendo notas y características comunes, cuenta con especialidades propias en cuanto a modalidad organizativa.

${ }^{41}$ GARCÍA NINET, J.I. (dir.), Lecciones sobre la Ley de Prevención de Riesgos Laborales, o.c., pp. 163-164. 


\subsection{Los servicios de prevención propios y mancomunados}

De acuerdo con el art. 15 RSP, el servicio de prevención propio constituye una unidad organizativa específica dentro de la empresa y debe incluir, como mínimo, dos de las especialidades o disciplinas preventivas de las recogidas en el art. 34 RSP (medicina del trabajo, seguridad en el trabajo, higiene industrial y ergonomía y psicosociología aplicada $)^{42}$. Aunque nada impide que en un mismo trabajador concurran diversas especialidades, debe considerarse necesario que para la constitución de un servicio de prevención propio han de ser varios los técnicos especialistas de nivel superior; y ello, porque el propio literal del art. 31.4 LPRL se refiere en plural a los "componentes de este servicio"43.

Sus integrantes, trabajadores de la empresa, deben dedicar de forma exclusiva su actividad en la misma a la finalidad de este servicio, y han de tener la capacitación requerida para las funciones que deban llevarse a cabo en los términos del capítulo VI RSP. Cuando el ámbito de actuación del servicio de prevención propio se extienda a más de un centro de trabajo, deberá tenerse en cuenta la situación de los diversos centros en relación con la ubicación del servicio, a fin de asegurar la adecuación de los medios de dicho servicio a los riesgos existentes (art. 15.3 RSP). Aquellas actividades preventivas no asumidas a través del servicio de prevención propio deberán ser concertadas con uno o más servicios de prevención ajenos.

La constitución de un servicio de prevención propio es obligatoria para el empresario cuando la empresa cuente con más de 500 trabajadores, o entre 250 y 500 trabajadores si realiza alguna de las actividades incluidas en el anexo I RSP (art. $14 \mathrm{RSP})^{44}$. A estos efectos, la unidad de cálculo la constituye la empresa

42 El servicio de prevención propio debe contar con medios humanos y materiales suficientes para poder cumplir su función preventiva, sin que resulte ajustada a derecho la organización de la prevención a través de la constitución meramente formal de un servicio de prevención propio al que no se le doten de los medios necesarios. STSJ Madrid, contenciosoadministrativo, 25 de julio de 2012 (n ${ }^{\circ}$ rec. 1999/2011).

${ }^{43}$ GARRIGUES GIMÉNEZ, A. (dir.), Derecho de la prevención de riesgos laborales, o.c., pp. 279-280. En contra, un sector de la doctrina ha entendido que un solo técnico con dos especialidades puede ser suficiente para conformar un servicio de prevención propio, pues nada señala la norma al respecto como sí hace en el caso de los servicios de prevención ajenos. Por todos, LUQUE PARRA, M., La organización de la prevención en la empresa, o.c., p. 85.

${ }^{44}$ También contempla este precepto un tercer supuesto de obligatoriedad de escasa concurrencia en la práctica: "Que, tratándose de empresas no incluidas en los apartados anteriores, así lo decida la autoridad laboral, previo informe de la Inspección de Trabajo y Seguridad Social y, en su caso, de los órganos técnicos en materia preventiva de las Comunidades Autónomas, en función de la peligrosidad de la actividad desarrollada o de la frecuencia o gravedad de la siniestralidad en la empresa, salvo que se opte por el concierto con una entidad especializada ajena a la empresa de conformidad con lo dispuesto en el artículo 16 de esta disposición". 
y no el centro de trabajo ${ }^{45}$. Deberán computarse todos los trabajadores existentes en la plantilla, con independencia del tipo de contrato que tengan o de la jornada laboral que desarrollen, tomando como referencia el periodo de un año ${ }^{46}$.

Respecto a la calificación de la actividad de la empresa, ha de seguirse el criterio interpretativo más favorable a la seguridad y salud laboral, por lo que, aunque su actividad principal no sea de anexo I RSP, deberá entenderse incluida en el mismo si parte de sus operaciones o procesos son subsumibles en las actividades incluidas en dicho anexo.

Como una subespecie de servicio de prevención propio, el art. 21 RSP regula los servicios de prevención mancomunados. El servicio de prevención mancomunado puede constituirse entre aquellas empresas que desarrollen simultáneamente actividades en un mismo centro de trabajo, edificio o centro comercial, siempre que quede garantizada la operatividad y eficacia del servicio para los diferentes centros de trabajo que lo constituyen. El concepto de servicio de prevención mancomunado implica que varias empresas se pongan de acuerdo y opten por esta modalidad preventiva, sin que resulte equiparable la mera adhesión de una empresa al servicio de prevención de otra ${ }^{47}$. Por negociación colectiva o mediante acuerdo o, en su defecto, por decisión de las empresas afectadas, podrá acordarse la constitución de servicios de prevención mancomunados entre aquellas empresas pertenecientes a un mismo sector productivo o grupo empresarial, o que desarrollen sus actividades en un polígono industrial o área geográfica limitada.

Las empresas que tengan obligación legal de disponer de un servicio de prevención propio no podrán formar parte de servicios de prevención mancomunados constituidos para las empresas de un determinado sector, aunque sí de los constituidos para empresas del mismo grupo. El acuerdo de constitución del servicio de prevención mancomunado se debe adoptar previa consulta a los representantes legales de los trabajadores de cada una de las empresas afectadas, haciendo constar expresamente las condiciones mínimas en que tal servicio de prevención debe desarrollarse (art. 21.2 RSP, en relación con el art. 33.1 b) LPRL). Los servicios de prevención mancomunados, tengan o no personalidad jurídica diferenciada, tendrán la consideración de servicios propios de las empresas que los constituyan y habrán de contar con, al menos, tres especialidades o disciplinas preventivas de las establecidas en el art. 34

\footnotetext{
${ }^{45}$ STSJ Madrid, contencioso-administrativo, 21 de octubre de 2005 ( ${ }^{\circ}$ rec. 542/2002).

${ }^{46}$ SALA FRANCO, T., Derecho de la Prevención de Riesgos Laborales, o.c., p. 158, y MORENO SOLANA, A., El servicio de prevención en la empresa. Modalidades de organización de la Prevención de Riesgos Laborales, o.c., pp. 273-281.

${ }^{47}$ STSJ Extremadura, 28 de febrero de 2005 ( $n^{\circ}$ rec. 394/2003).
} 
RSP. Para poder constituirse deberán disponer de los recursos humanos y materiales mínimos equivalentes a los exigidos para los servicios de prevención ajenos de acuerdo con lo establecido en el RSP y en su normativa de desarrollo.

\subsection{Los servicios de prevención ajenos y su cuestionada capacidad de integración}

Cuando la empresa no lleve a cabo las actividades preventivas con recursos propios (asunción del empresario, designación de trabajadores, servicio de prevención propio o mancomunado), la organización de las funciones en materia preventiva solo podrá hacerse a través de un servicio de prevención ajeno debidamente acreditado (art. 31.3 LPRL). El servicio de prevención ajeno es una entidad especializada externa e independiente de la empresa, que concierta con la misma la realización de actividades de prevención y el asesoramiento y apoyo que precise en función de los tipos de riesgos.

Los servicios de prevención ajenos se regulan en el art. 31 LPRL y en los arts. 16 y ss. RSP. Cualquier empresa puede concertar la totalidad de la actividad preventiva con uno o varios servicios de prevención ajenos, salvo que esté obligada a constituir un servicio de prevención propio. También podrá concertarse un servicio de prevención ajeno parcialmente cuando el empresario, los trabajadores designados o el servicio de prevención propio o mancomunado, no asuman todas las especialidades preventivas; en este caso, el servicio de prevención ajeno dará cobertura a las disciplinas preventivas que no hayan sido asumidas con medios propios. De acuerdo con el art. 33.1 b) LPRL, los representantes de los trabajadores deberán ser consultados por el empresario con carácter previo a la adopción de la decisión de concertar la actividad preventiva con uno o varios servicios de prevención ajenos. Como ya se ha anotado más arriba, esta consulta tiene carácter preceptivo, pero no resulta vinculante para el empresario.

Para actuar como servicios de prevención ajenos las entidades especializadas deberán ser objeto de una acreditación por la autoridad laboral, que será única y con validez en todo el territorio español, mediante la comprobación de que reúnen los requisitos que se establezcan reglamentariamente y previa aprobación de la autoridad sanitaria en cuanto a los aspectos de carácter sanitario (art. 31.5 LPRL). Desarrollando este extremo, el art. 18 RSP determina que las entidades especializadas deben contar con las instalaciones y los recursos materiales y humanos que les permitan ejecutar adecuadamente la actividad preventiva que hubieren concertado, teniendo en cuenta el tipo, extensión y frecuencia de los servicios preventivos que han de prestar, el tipo de actividad desarrollada por los trabajadores de las empresas concertadas, y la ubicación y tamaño de los centros de trabajo en los que dicha prestación ha de llevarse a cabo. 
Entre otros requisitos, se exige el de disponer como mínimo de un técnico que ostente la cualificación necesaria para el desempeño de las funciones de nivel superior de acuerdo con lo establecido en el capítulo VI RSP por cada una de las especialidades o disciplinas preventivas, salvo en el caso de la especialidad de medicina del trabajo que exigirá contar, al menos, con un médico especialista en medicina del trabajo o diplomado en medicina de empresa y con un enfermero de empresa. Asimismo, deberán disponer del personal necesario que tenga la capacitación requerida para desarrollar las funciones de los niveles básico e intermedio previstas en el RSP en atención a las características de las empresas cubiertas por el servicio ${ }^{48}$.

Gran parte de la doctrina iuslaboralista aboga por considerar subsidiaria esta modalidad organizativa, estimando que ha de emplearse con carácter meramente complementario a las modalidades internas, que son claramente preferentes en atención al "principio de proximidad del diseño y control preventivo respecto a los factores y fuentes de riesgos en la organización productiva",49. Estas interpretaciones identifican integración con internalización, y por ello consideran que solo mediante el proceso de internalización de los servicios de prevención será posible conseguir la ansiada integración de la prevención en el seno de la empresa.

No parece esta una premisa a la que se la pueda otorgar validez absoluta desde un punto de vista empírico a la vista de los estudios de campo desarrollados; ni toda internalización implica integración, ni la contratación de un servicio de prevención ajeno supone necesariamente laminar el principio de integración preventiva $^{50}$. Para que la integración preventiva se produzca, necesariamente

${ }^{48}$ Requisitos que han sido precisados en el ámbito técnico por la Orden TIN/2504/2010, de 20 de septiembre, por la que se desarrolla el RSP, en lo referente a la acreditación de entidades especializadas como servicios de prevención, memoria de actividades preventivas y autorización para realizar la actividad de auditoría del sistema de prevención de las empresas; y en el ámbito sanitario por el RD 843/2011, de 17 de junio, por el que se establecen los criterios básicos sobre la organización de recursos para desarrollar la actividad sanitaria de los servicios de prevención.

${ }^{49}$ MONEREO PÉREZ, J.L., y LÓPEZ INSUA, B.M., "El derecho a la prevención: un análisis del modelo organizativo preventivo español", en AAVV, Ley de Prevención de Riesgos Laborales. Una revisión crítica, veinte años después, Madrid 2016, p. 90. En idéntico sentido, BLASCO MAYOR, A., "La externalización de las actividades de prevención de riesgos laborales", en Aranzadi Social, 5 (2001) 909-926, y MOLINA HERMOSILLA, O., "El mercado de los servicios de prevención: la búsqueda del difícil equilibrio entre el cumplimiento de una función social y la obtención de un beneficio empresarial”, en Revista de Derecho Social, 48 (2009) 134-143.

50 Vid. el ilustrativo estudio de BERBEGAL-MIRAVENT, J., y CANNTONET JORDI, Ma. L., "La tendencia hacia la servitización en la gestión de la prevención de riesgos laborales", en Intangible Capital, vol. 10, 2 (2014) 317-341. Cfr. INSST, La gestión preventiva en las empresas en España. Análisis del módulo de prevención de riesgos laborales de la "Encuesta anual laboral 2016", Madrid 2018. 
hará falta la concurrencia de dos elementos: una modalidad preventiva de calidad -ya sea con medios propios o ajenos- y una empresa con un compromiso real con la prevención de riesgos laborales. No es posible hacer recaer todas las insuficiencias de la integración de la prevención en el tipo de modalidad preventiva escogida, pues ello supone desconocer la trascendencia de la otra variable señalada, que tiene igual o más importancia que la primera.

Con independencia del valor que estas y otras construcciones doctrinales puedan tener desde un plano estrictamente teórico, lo cierto es que la realidad productiva de nuestro país, marcada por las pequeñas y medianas empresas con escaso volumen de trabajadores, ha hecho que los servicios de prevención ajenos se erijan en la modalidad organizativa más empleada por las organizaciones productivas, tal y como se desprende de los diferentes estudios realizados sobre la materia ${ }^{51}$.

\section{A MODO DE REFLEXIÓN FINAL}

Desde la entrada en vigor de la LPRL, la organización de la prevención ha sido objeto de una especial atención doctrinal; atención que se ha mantenido constante hasta nuestros días, teniendo en cuenta el carácter transversal de la materia, que imbrica la dinámica del conjunto de obligaciones preventivas empresariales.

Las distintas modalidades de organización preventiva que diseña la LPRL han sido uno de los extremos más cuestionados de nuestra regulación preventiva ${ }^{52}$. Los desajustes y disfunciones que en la práctica ha suscitado la ordenación legal del deber empresarial de organizar la prevención se han visto corregidos, aunque de forma limitada e insuficiente, por las sucesivas reformas legislativas que han afectado a esta materia ${ }^{53}$.

${ }^{51}$ En el ámbito estatal, de los últimos datos existentes se deriva que un $78 \%$ de las empresas recurren a un servicio de prevención ajeno como modelo de gestión preventiva. Vid. INSST, Encuesta Nacional de Gestión de Riesgos Laborales en las Empresas. ESENER-2 España, $o p$. cit. Este dato refleja que la tendencia a organizar la prevención de riesgos laborales a través de servicios de prevención ajenos no solo no disminuye, sino que aumenta en comparación con datos anteriores en los que este porcentaje alcanzaba el 73\%. Cfr. INSST, Encuesta Nacional de Gestión de la Seguridad y Salud en las Empresas, Madrid 2009.

${ }^{52} \mathrm{Vid}$. MORENO SOLANA, A., "El modelo actual de organización de la prevención en las empresas en el debate político", en Trabajo y Derecho, 21 (2016) 123-134.

${ }^{53}$ Entre otras, resultan especialmente destacables la Ley 54/2003, de 12 de diciembre, de reforma del marco normativo de la prevención de riesgos laborales, y el RD 337/2010, de 19 de marzo, por el que se modifican, entre otras normas, el RSP. 
En todas estas reformas normativas se ha perseguido fomentar la internalización de la prevención, diseñando un marco regulatorio más propicio para facilitar la asunción empresarial por el empresario o el empleo de medios propios en la gestión preventiva frente al recurso a servicios externos. Se pretendía con estas modificaciones legislativas variar la configuración de los modelos organizativos escogidos libremente por las empresas, fomentando el empleo de los medios internos frente a los externos; y ello, sin alterar los pilares básicos sobre los que se asentó la organización de la prevención en la configuración normativa originaria ${ }^{54}$.

Sin embargo, la timidez de las modificaciones y su carácter adjetivo se han mostrado totalmente insuficientes para lograr los objetivos perseguidos. En este sentido, la intención pedagógica del legislador no se ha visto acompañada en la práctica por un cambio de tendencia en las opciones empresariales en relación con la organización de la prevención. La realidad productiva evidencia que las empresas siguen inclinándose por el recurso a servicios externos para organizar la prevención, por lo que las reformas normativas que se han ido sucediendo se presentan de todo punto ineficaces para alcanzar el cambio en la tendencia de externalización de la gestión preventiva pretendido inicialmente por el legislador.

Los nuevos retos que la digitalización y las nuevas formas de organización del trabajo presentan hacen necesario un replanteamiento de la seguridad y salud laboral, debiendo también alcanzar a los modelos de gestión preventiva actualmente existentes ${ }^{55}$. Entre otros extremos, la efectiva integración de la prevención en el seno de las empresas se presenta como uno de los desafíos más importantes que nuestro sistema jurídico ha de acometer de forma decidida, teniendo en cuenta el déficit que nuestro modelo productivo presenta en relación con este extremo. En esta tarea, el legislador ha de huir de prejuicios y posturas dogmáticas que identifican la integración con la internalización. Como se ha señalado, ni toda internalización implica integración, ni la externalización supone necesariamente laminar el principio de integración preventiva.

La anhelada integración preventiva requiere, indudablemente, una modalidad preventiva de calidad, ya sea con medios propios o ajenos, pero también de empresas comprometidas realmente con la prevención de riesgos laborales y

${ }^{54}$ IGARTUA MIRÓ, Ma . T., "Organización de la prevención en la empresa: de los excesos de la externalización al tímido fomento del uso de recursos propios. Reflexiones críticas al hilo de dos disposiciones recientes (RD 337/2010 y RD 404/2010)", en Documentación Laboral, 88 (2010) 93.

${ }^{55}$ Cfr. MELLA MÉNDEZ, L., "Los retos de la prevención de riesgos laborales ante la digitalización de la empresa y las nuevas formas de trabajo: puntos críticos", en Revista Española de Derecho del Trabajo, 229 (2020), y MERCADER UGUINA, J. R., "Riesgos laborales y transformación digital: hacia una empresa tecnológicamente responsable", en Teoría y Derecho: revista de pensamiento jurídico, 23 (2018). 
con la cultura preventiva. Incidir solo sobre una de las variables, el tipo de modalidad preventiva, supone desconocer la trascendencia de la otra variable señalada, que tiene igual o más importancia que la primera. Insistir en modulaciones normativas que afecten a los sistemas de organización preventiva, sin implementar políticas públicas transversales que consigan integrar la cultura preventiva en la conciencia colectiva, parece que solo conducirá a soluciones parciales y fragmentarias que, como la experiencia ha demostrado, se demuestran de todo punto ineficaces para variar la concepción comunitaria de la seguridad y salud laboral.

\section{BIBLIOGRAFÍA}

- BERBEGAL-MIRAVENT, J., y CANNTONET JORDI, Ma. L., "La tendencia hacia la servitización en la gestión de la prevención de riesgos laborales", en Intangible Capital, vol. 10, 2 (2014).

- BLASCO MAYOR, A., "La externalización de las actividades de prevención de riesgos laborales", en Aranzadi Social, 5 (2001).

- FERNÁNDEZ MARCOS, L., Comentarios a la Ley de Prevención de Riesgos Laborales y normativa complementaria, Madrid 2001.

- FERNÁNDEZ MARCOS, L., "El contenido de la Ley de Prevención de Riesgos Laborales: Puntos críticos", en Actualidad Laboral, 3 (1995).

- GARATE CASTRO, J., "Organización de la prevención mediante la designación de uno o varios trabajadores", en Revista General de Derecho del Trabajo y de la Seguridad Social, 4 (2003).

- GARCÍA GONZÁLEZ, G. y GARRIGUES GIMÉNEZ, A. (dirs.), Manual de Derecho de la Prevención de Riesgos Laborales, Valencia 2015.

- GARCÍA NINET, J.I. (dir.), Lecciones sobre la Ley de Prevención de Riesgos Laborales, Castelló de la Plana 1997.

- GARRIGUES GIMÉNEZ, A. (dir.), Derecho de la prevención de riesgos laborales, Albacete 2009.

- GONZÁLEZ ORTEGA, S., "La aplicación de la Ley de Prevención de Riesgos Laborales a las administraciones públicas", en Temas Laborales, 72 (2003). 
- GONZÁLEZ ORTEGA, S., "La organización de la prevención por el empresario: los servicios de prevención", en Temas Laborales, 50 (1999).

- GONZÁlEZ ORTEGA, S., y APARICIO TOVAR, J., Comentarios a la Ley 31/1995 de Prevención de Riesgos Laborales, Madrid 1996.

- IGARTUA MIRÓ, Ma.T., "Organización de la prevención en la empresa: de los excesos de la externalización al tímido fomento del uso de recursos propios. Reflexiones críticas al hilo de dos disposiciones recientes (RD 337/2010 y RD 404/2010)", en Documentación Laboral, 88 (2010).

- INSST, Encuesta Nacional de Gestión de la Seguridad y Salud en las Empresas, Madrid 2009.

- INSST, Encuesta Nacional de Gestión de Riesgos Laborales en las Empresas. ESENER-2 España, Madrid 2015.

- INSST, La gestión preventiva en las empresas en España. Análisis del módulo de prevención de riesgos laborales de la "Encuesta anual laboral 2016" “, Madrid 2018.

- LOUSADA AROCHENA, J.F., "Prevención de riesgos laborales en pequeñas y medianas empresas", en Revista Española de Derecho del Trabajo, 171 (2014).

- LUQUE PARRA, M., La organización de la prevención en la empresa, Valencia 2001.

- MARTÍNEZ ASO, M., La eficacia de la protección del derecho a la seguridad y salud en el trabajo de los trabajadores extranjeros, Girona 2013. Disponible en:https://www.tdx.cat/bitstream/handle/10803/124038/tmma.pdf;sequence=2

- MELLA MÉNDEZ, L., "Los retos de la prevención de riesgos laborales ante la digitalización de la empresa y las nuevas formas de trabajo: puntos críticos”, en Revista Española de Derecho del Trabajo, 229 (2020).

- MERCADER UGUINA, J. R., "El Reglamento de los Servicios de Prevención: Crónica de una norma largamente esperada", en Relaciones Laborales, 1 (1997).

- MERCADER UGUINA, J. R., "La Ley de Prevención de Riesgos Laborales, veinte años después", en Revista de Información Laboral, 8 (2015). 
- MERCADER UGUINA, J. R., "Ley de Prevención de Riesgos Laborales y Administraciones Públicas", en Relaciones Laborales, 1 (1996).

- MERCADER UGUINA, J. R., "Riesgos laborales y transformación digital: hacia una empresa tecnológicamente responsable", en Teoría y Derecho: revista de pensamiento jurídico, 23 (2018).

- MOLINA HERMOSILLA, O., "El mercado de los servicios de prevención: la búsqueda del difícil equilibrio entre el cumplimiento de una función social y la obtención de un beneficio empresarial", en Revista de Derecho Social, 48 (2009).

- MOLINA NAVARRETE, C., "Servicios de Prevención", en AAVV, Comentario a la Ley de Prevención de Riesgos Laborales y sus desarrollos reglamentarios, Granada 2004.

- MONEREO PÉREZ, J.L., y LÓPEZ INSUA, B.M., "El derecho a la prevención: un análisis del modelo organizativo preventivo español", en AAVV, Ley de Prevención de Riesgos Laborales. Una revisión crítica, veinte años después, Madrid 2016.

- MONTOYA MELGAR, A., "Prólogo", en GUTIÉRREZ-SOLAR CALVO, B., El deber de seguridad y salud en el trabajo. Un estudio sobre su naturaleza jurídica, Madrid 1999.

- MONTOYA MELGAR, A., y PIZÁ GRANADOS, J., Curso de seguridad y salud en el trabajo, Madrid 2000.

- MORENO SOLANA, A., "El modelo actual de organización de la prevención en las empresas en el debate político", en Trabajo y Derecho, 21 (2016).

- MORENO SOLANA, A., El servicio de prevención en la empresa. Modalidades de organización de la Prevención de Riesgos Laborales, Cizur Menor 2016.

- OJEDA AVILÉS, A., "Claves interpretativas de la Ley 31/1995, sobre prevención de riesgos laborales", en La Ley, 1 (1997).

- ROMERAL HERNÁNDEZ, J., "Derechos de información, consulta y crédito horario del delegado de prevención a la luz de la jurisprudencia", en Revista Española de Derecho del Trabajo, 209 (2018).

- SALA FRAnCO, T., Derecho de la Prevención de Riesgos Laborales, Valencia 2011. 
- SEMPERE NAVARRO, A.V., "Aspectos laborales de las leyes sobre libre acceso a los servicios", en Aranzadi Doctrinal, 2 (2010).

- SEMPERE NAVARRO, A.V., "Aspectos jurídicos del Reglamento de Prevención de Riesgos Laborales”, en Aranzadi Social, 5 (1997).

- TOSCANI GIMÉNEZ, D., "Obligaciones y derechos de los trabajadores con funciones preventivas", en Gestión Práctica de Riesgos Laborales, 87 (2011).

- TUDELA CAMBRONERO, G., y VALDEOLIVAS GARCÍA, Y., La seguridad y la salud laboral en la negociación colectiva, Madrid 2009.

- VALLEJO DACOSTA, R., y LAFUENTE PASTOR, V.P., Marco jurídico de la seguridad y salud en el trabajo, Zaragoza 2016.

- VIDA SORIA, J. (dir.), Manual para la formación en prevención de riesgos laborales, Valladolid 2003. 